\title{
INTERACTION OF CHROMOPHORES IN MONOLAYER ASSEMBLIES
}

\author{
HANS KUHN
}

\begin{abstract}
Max-Planck-Institut für Biophysikalische Chemie, Karl-Friedrich-BonhoefferInstitut, D 3400 Göttingen, Germany
\end{abstract}

\begin{abstract}
The techniques of producing monolayer assemblies are discussed. In the present context these assemblies are of interest because of the interactions between chromophores included in the monolayers-particularly those interactions which lead to energy transfer between the chromophores. Fluorescence effects resulting from this transfer can be utilized to monitor the manipulation of the monolayers and to elucidate the architecture of various types of the assemblies, including those in which there are included protein monolayers.

The fact that it is possible to control the interchromophore distance in the assemblies enables quantitative determination of the distance-dependence of the energy transfer process; by comparison with theory it is then possible to elucidate the multipole nature of the spectroscopic transitions involved. The energy transfer results in changes in lifetimes which can be interpreted quantitatively. A new effect in luminescence is discussed and demonstrated by the monolayer assembling technique (the dependence of the lifetime of an excited molecule surrounded by a non-absorbing dielectric on details in its broad environment).

Application is also made to molecular aggregates i.e. structures in which the chromophores are in direct contact. In the case of sandwich-pairs the shift in absorption peak is in quantitative agreement with that calculated from the electron-gas model. Consideration of the shifts in Scheibe-type aggregates leads to the development of a new structural model-the 'brick stonework' model-for these aggregates.

This model has enabled assembling monolayer structures which show the Scheibe-type effect. Dipole moment and polarizability of the excited state of the aggregate are calculated and measured using a new type of electrochromism, obtained by sandwiching a single monolayer of the aggregated dye between fatty acid layers and semitransmitting metal electrodes.
\end{abstract}

The spectroscopic study of chromophore interactions in monolayer assemblies has two aspects:

Investigating monolayers and investigating the nature of interactions.

In this paper we discuss recently investigated examples concerning the first aspect (section 1) and the second aspect (sections 2-3).

\section{ENERGY-TRANSFER MONITORED MANIPULATION OF MONOLAYERS}

(a) Separation and re-assembling monolayers

The energy transfer between a sensitizer dye in a monolayer and an 


\section{HANS KUHN}

acceptor dye in a monolayer deposited on to the first may be easily investigated by studying the fluorescence quenching of the sensitizer or the sensitized fluorescence of the acceptor ${ }^{1,2}$. It should be easy then, by energy transfer, to study the separation and reassembling of monolayers. The exciting problem of a controlled manipulation of objects in the range of molecular dimensions may thus be attacked. An energy-transfer monitored separation of monolayers may be realized in the following way ${ }^{3,} \uparrow$ :

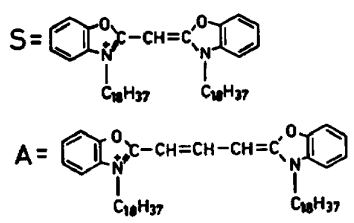

(a)
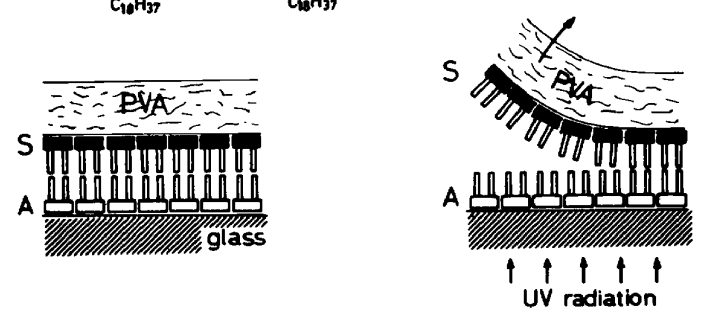

(b)
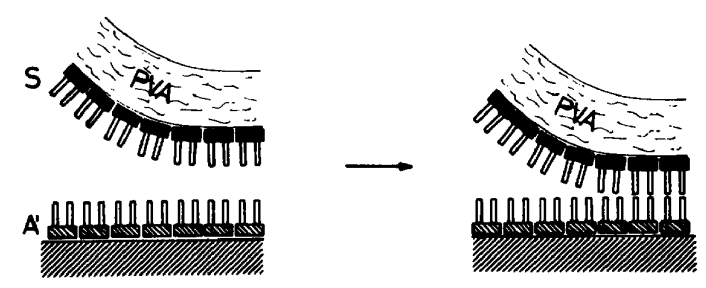

(c)
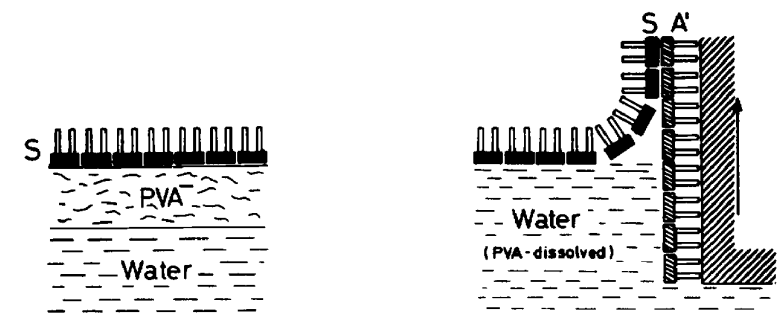

Figure 1. (a) Separation of monolayer S (sensitizer) and A (acceptor); (b) contacting monolayer $S$ (side with hydrocarbon chain ends) with monolayer $A^{\prime}$; (c) contacting monolayer $S$ (side with chromophores) with monolayer $\mathbf{A}^{\prime}$. The monolayers (mixed monolayers of stearylsubstituted dye and arachidic acid, in molar mixing ratio $1: 10$ ) are represented schematically, omitting arachidic acid molecules. The chains of the fatty acid molecules and the hydrocarbon substituents of the dye molecules are tightly packed in the layer and oriented parallel to each other and perpendicular to the layer plane.

$\dagger$ Rothen $^{4}$ reported a separation of monolayers to occur within molecular accuracy in systems of proteins and lipids, when using a Scotch Tape stripping technique ("molecular microtome'). 


\section{CHROMOPHORES IN MONOLAYER ASSEMBLIES}

Mixed monolayers of dyes S and A (Figure 1) and arachidic acid, which have a well defined and compact structure ${ }^{2}$, are deposited on a glass plate according to Figure 1 and a polyvinylalcohol film superposed. The film is stripped off as shown in Figure $1 a$ and it may be demonstrated that the separation occurs between the two layers:

In the part to the right the two layers are in contact on the plate. When irradiating with UV light, which is absorbed by $S$, the blue fluorescence of $S$ is largely quenched and the yellow fluorescence of A is observed. In the part on the left the sensitizer layer is stripped off. The film shows the strong blue fluorescence of $S$, demonstrating the S-layer to be stripped off with the film. The glass plate with layer A appears black since A does not absorb the incident radiation and thus does not fluoresce. However, when we excite with light absorbed by $\mathrm{A}$ a uniform yellow fluorescence of the glass plate in both parts is observed showing that a layer is present on the glass plate where the S-layer is stripped off ${ }^{3}$.

By setting the stripped film back onto the plate a reassembling of the dye monolayers in the molecular scale is possible (Figure 1b): the fluorescence of $S$ is quenched and the sensitized fluorescence of $A$ is observed at the touching parts, which appear like the part where the polymer film has not been stripped off, demonstrating a molecular contact of the layers. The layer fixed at the polymer film may be brought into contact with any other monolayer $\mathrm{A}^{\prime}$ on a support. Again the molecular contact is indicated by the appearance of the sensitized fluorescence. In this example the neighbour molecules on one side of the monolayer of dye $\mathrm{S}$ were changed.

It is also possible to change, in a controlled way, the molecules attaching to the second side of the same monolayer. The polymer film is dissolved according to Figure $1 c$ and then the second side of the polymer is brought into contact with an appropriate acceptor. Again a fluorescence quenching
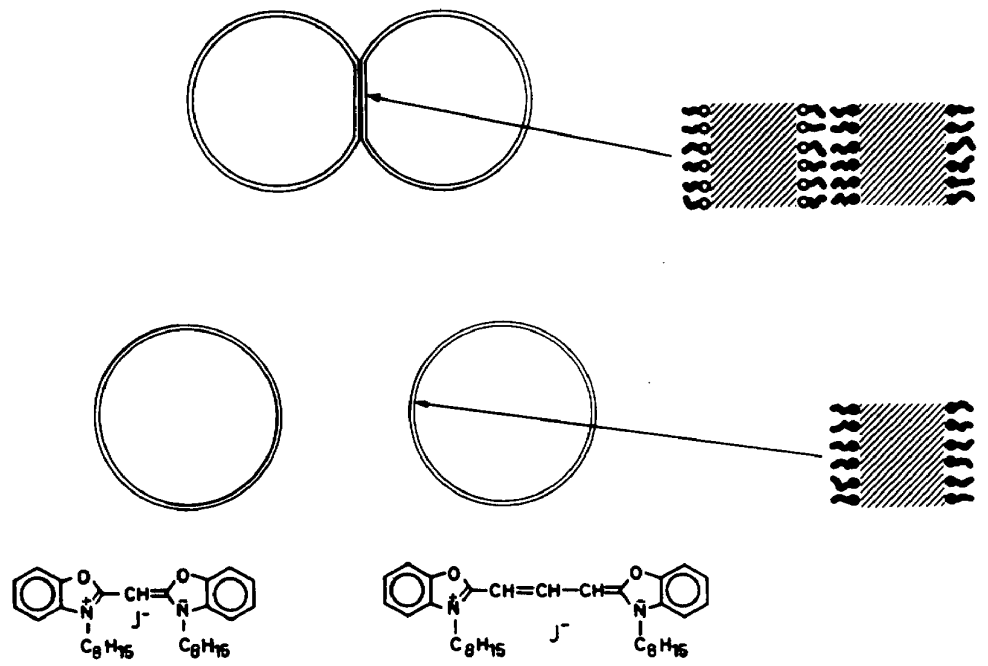

Figure 2. Contacting and separation of soap bubbles. Justification of assumed membrane architecture in contact area by using surface active fluorescing dyes. 
of $\mathrm{S}$ and a sensitized fluorescence of $\mathrm{A}$ is observed showing that a molecular contact at this second side of the monolayer of $\mathbf{S}$ can also be achieved ${ }^{3}$.

These experiments demonstrate how easily monolayers can be manipulated. The possibility of constructing monolayer assemblies of any complicated architecture on a glass plate, of bringing it onto a water surface and of transferring it to any appropriate substrate may be useful in building organized systems by assembling prefabricated elements.

The controlled joining and removal of monolayers may also be achieved by bringing soap bubbles into contact and subsequently separating them (Figure 2) ${ }^{5}$. Surface active fluorescent dyes incorporated in the surface layers of the bubbles are not transferred from one bubble to the other showing that the monolayers, when brought into contact, do not rearrange and that the dye molecules are not exchanged between the touching monolayers. In this way a new possibility of forming bimolecular lipid membranes is obtained. Unlike the membranes obtained by the known techniques ${ }^{6}$ these membranes may be made asymmetric.

\section{(b) Protein Monolayers}

In the case of protein monolayers the manipulation and construction of organized assemblies is of particular interest regarding their implications for a synthetic molecular biology. Several proteins have been adsorbed in an enzymatically active form at a monolayer film of arachidic-acid or methylstearate $^{\tau}$. In this way compact layers of the proteins are obtained. This is best seen from the electron micrograph of the iron rich protein ferritine

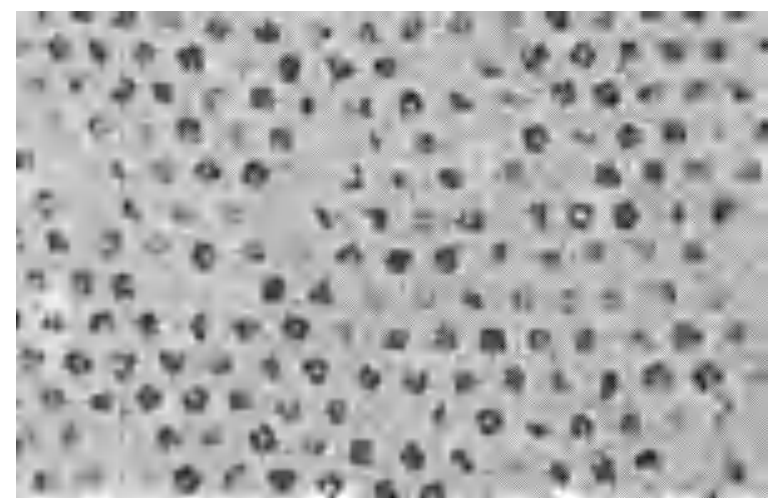

Figure 3. Electron micrograph of protein ferritine adsorbed on a monolayer of methylstearate and trimethyldodecylammonium bromide. Protein molecules form monolayer with long range order?

(Figure 3). These films, handled like fatty acid films, may be used as components of organized assemblies of monolayers. We discuss a possibility for proving the architecture of an assembly by studying energy transfer from a dye to a chromophoric group in the protein ${ }^{7}$. An assembly with the architecture shown in Figure $4 a$ may be easily obtained following the usual 


\section{CHROMOPHORES IN MONOLAYER ASSEMBLIES}

procedure. The haem-groups of the protein act as acceptors for the excitation energy of dye $S$ and quench its fluorescence. The fluorescence being a measure of the distance between sensitizer and acceptor, the architecture of the system may be proved. The fluorescence intensity is plotted against an
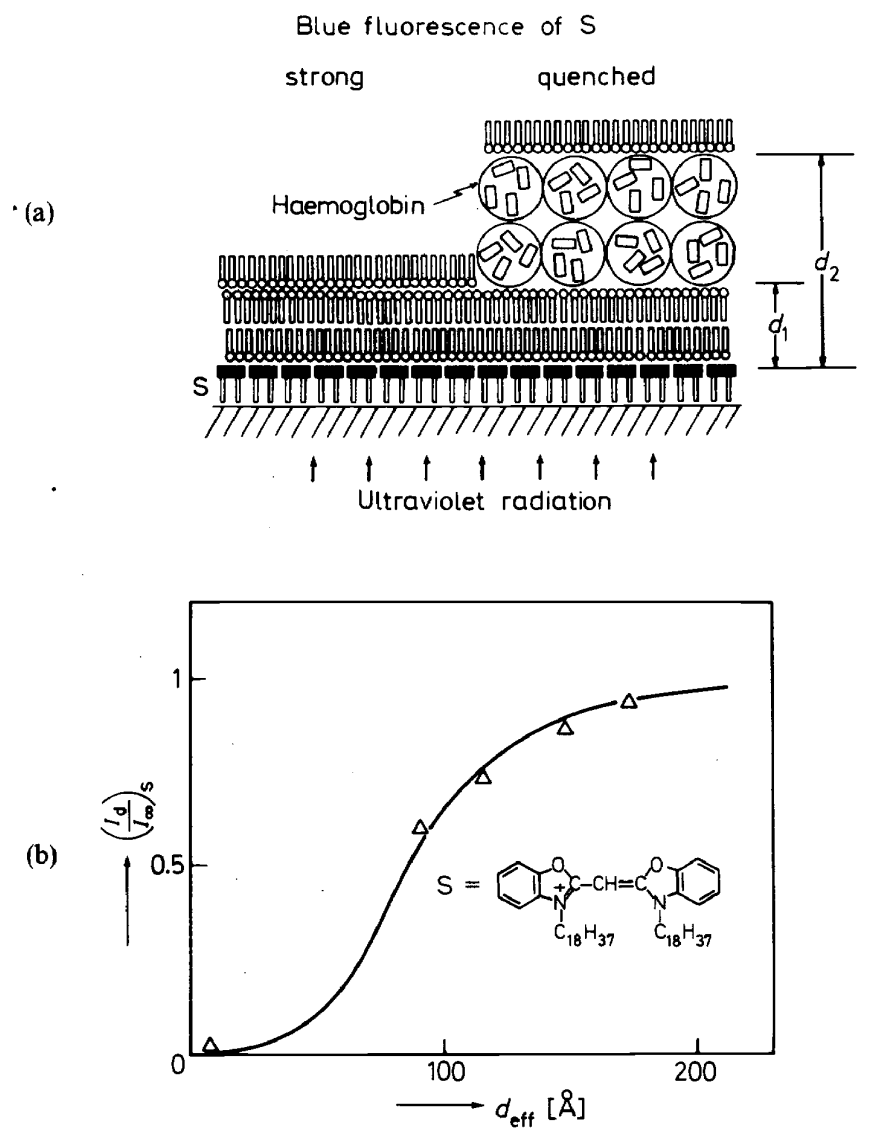

Figure 4. Energy transfer from fluorescing dye $\mathrm{S}$ to haemoglobin. (a) Cross section of arrangement. (b) Intensity of fluorescence of $S$ against $d_{\text {eff }}$, where $1 / d_{\text {eff }}^{4}=\left(\frac{1}{3}\right)\left(d_{1}^{-3}-d_{2}^{-3}\right) /\left(d_{2}-d_{1}\right)$; experimental points and theoretical curve.

appropriately defined effective distance $d_{\text {eff }}$ (Figure $4 b$ ). The experimental points lie on the theoretical curve. A monolayer of plasma-albumin was interposed between sensitizer dye and acceptor haemoglobin ${ }^{7}$. The pronounced increase in fluorescence intensity is a measure of the thickness of this additional interlayer. Its value agrees with expectation. This demonstrates the possibility of assembling complicated arrangements containing different proteins. 


\section{NATURE OF DEACTIVATION PROCESSES}

\section{(a) Multipole nature of phosphorescence of dyes}

It is well known that the fluorescence of a molecule corresponds to a dipole emission, but little is known about the multipole nature of a forbidden transition such as phosphorescence ${ }^{8}$. There a molecule might act as an electric quadrupole, magnetic dipole or electric dipole emitter. In all three cases a long range energy transfer must be expected, the distance-dependence being different in each case ${ }^{9}$. Thus, by comparing the experimental distancedependence of energy transfer with the theoretical dependence found for each case, the multipole nature of the phosphorescence may be determined. Since it was assumed before that an energy transfer over a large range is only possible for dipole-dipole interaction, this point shall be discussed in more detail.

For describing the luminescence and the energy transfer it is advantageous to use the classical approach. The results, very easily obtained and translated into quantum mechanical language, agree with those obtained by a more strict treatment ${ }^{9}$.

The molecule is described as a classical antenna. The emission power $L_{e}$ is obtained from the classical electromagnetic theory and is given in Table 1

Table 1 . Power emitted and absorbed for various multipole radiations $\mu_{0}, m_{0}, Q_{0}$, amplitude of electric dipole, magnetic dipole and electric quadrupole respectively; $c$ the velocity of light, $\omega=2 \pi v_{s}, \sigma$ number of molecules per unit area, $\alpha$ a numerical factor discussed following equation

(4)

\begin{tabular}{|c|c|c|c|}
\hline Emitter & Magnetic dipole & Electric dipole & electric quadrupole \\
\hline$L_{e}$ emitted power & $\left(1 / 48 \pi^{2} c\right) m_{0}^{2} \omega^{4}$ & $\left(1 / 3 c^{3}\right) \mu_{0}^{2} \omega^{4}$ & $\left(1 / 60 c^{5}\right) Q_{0}^{2} \omega^{6}$ \\
\hline $\begin{array}{l}L_{\mathrm{a}} \text { power absorbed } \\
\text { by acceptor layer }\end{array}$ & const. $\times \omega^{2} \times m_{0}^{2} a \sigma \times \frac{1}{d^{2}}$ & const. $\times \mu_{0}^{2} a \sigma \times \frac{1}{d^{4}}$ & const. $\times Q_{0}^{2} a \sigma \times \frac{1}{d^{6}}$ \\
\hline at distance $d$ & const. $=\frac{2 \pi}{3} \alpha^{2}$ & const. $=\frac{128 \pi^{5}}{3} \alpha^{4}$ & const. $=\frac{128 \pi^{7}}{15} \alpha^{6}$ \\
\hline
\end{tabular}

for the three cases. The probability of an excited molecule emitting a quantum of light (energy $h v_{s}$ ), in the time interval $\mathrm{d} t$, is $\mathrm{d} P_{e}$ and is given by

$$
\frac{\mathrm{d} P_{e}}{\mathrm{~d} t}=\frac{L_{e}}{h v_{\mathrm{s}}}
$$

Now let us put an acceptor molecule at a point near the emitting antenna. In the classical picture the emitting molecule produces a certain alternating electromagnetic field, and $F_{0}$ shall be the amplitude of the electric field strength. The power absorbed by the acceptor molecule is

$$
L_{a}^{\prime}=a F_{0}{ }^{2}
$$

where constant $a$ depends on the nature of the acceptor molecule and is related to the extinction coefficient of the molecule for light of frequency $v_{s}$.

The field $F_{0}$ in the environment of an oscillating multipole is given by Hertz's solution of the Maxwell equations and in the proximity field region 
(at a distance small compared to the wavelength of the luminescent light $\lambda_{s}$ ), $F_{0}$ is simply given by Coulomb's law and the induction laws for electric and magnetic multipoles respectively (Figure 5 ). The power absorbed by a layer

$$
\begin{aligned}
& \longmapsto \\
& \mu_{0} \overbrace{}^{\rho} \quad \downarrow F_{0}=\frac{\mu_{0}}{r^{3}} \\
& m_{0}\left[\begin{array}{l}
S \\
N
\end{array} \quad \cdot F_{0}=\frac{m_{0} \omega}{r^{2}}\right. \\
& Q_{0} \odot \oplus \oplus \\
& \longrightarrow F_{0}=\frac{3 Q_{0}}{r^{4}}
\end{aligned}
$$

Figure 5. Amplitude of electric field $\left(F_{0}\right)$ in proximity of oscillating electric dipole (amplitude $\mu_{0}$ ). magnetic dipole (amplitude $m_{0}$ ), electric quadrupole (amplitude $Q_{0}$ ).

of acceptor molecules, $L_{a}$, is obtained by summing over the contributions $a F_{0}{ }^{2}$ of each single molecule (Table 1 ).

Energy transfer and emission being competitive processes, the lifetime of the excited state is shortened by increasing the amount of energy transfer, and thus the decay time of the phosphorescence is shortened. The decay time $\tau_{d}$ for a distance $d$ between sensitizer and acceptor layer is related to $L_{a}, L_{e}$ and $L_{t}\left(L_{t}\right.$ is the power loss by non-radiative processes, other than the process of energy transfer to acceptor A):

$$
\tau_{d} / \tau_{\infty}=\left(L_{e}+L_{t}\right) /\left(L_{e}+L_{t}+L_{a}\right) .
$$

The quantum yield of the luminescence, $q_{s}$ is related to $L_{e}$ and $L_{t}$ by $q_{s}=L_{e} /\left(L_{e}+L_{t}\right)$. [When A is absent, $q_{s}$ is the probability of a molecule in the luminescent excited state emitting a photon. $q_{s}$ must be distinguished from $q_{s}^{\prime}$ (number of emitted quanta/number of absorbed quanta)]. With the data in Table 1 the equation

$$
\begin{aligned}
\frac{\tau_{d}}{\tau_{\infty}} & =\left[1+\left(d_{0} / d\right)^{p}\right]^{-1} \\
d_{0} & =\alpha\left(\lambda_{s} / n\right)\left(A_{s} q_{s}\right)^{1 / p}
\end{aligned}
$$

is obtained with $p=2,4$ and 6 for magnetic dipole, electric dipole, and electric quadrupole, respectively ${ }^{10} . n$ is the refractive index of the medium. The value of the numerical factor $\alpha$ depends on the orientation of emitter and acceptor. Under usual experimental conditions the transition moments of the acceptor molecules are statistically oriented in the plane of the layer. Then $\alpha=3^{\frac{1}{2}} / 8 \pi$ for a magnetic dipole and for an electric dipole $\alpha$ ranges between the values 0.098 and 0.12 , for an electric quadrupole between the values 0.14 and 0.21 . In the case of a sufficiently narrow luminescence band 
$A_{s}$ is the absorption (1-transmission) of the acceptor layer. In the general case, $A_{s}$ is defined by the expression

$$
A_{s}=\int A(v) f_{s}(v)\left(v_{s} / v\right)^{4} \mathrm{~d} v
$$

with $f_{s}(v)$ being the normalized quanta distribution function, and $v_{s}$ the frequency of the maximum of the fluorescence band of the sensitizer; $A(v)$ is the absorption of $A$ at frequency $v$. The value of the quantum yield, $q_{s}$, must lie between 1 and 0.06 since the measured overall quantum yield $q_{s}^{\prime}$ (number of emitted quanta/number of absorbed quanta) is 0.06 . By inserting the lower limit of $q_{s}$ and $\alpha$ and the upper limit of $q_{s}$ and $\alpha$ in equation (4) we find the results given in Figure 6. The experimental points are given in the figure. The experimental value $d_{0}=100 \AA$ rules out an electric quadrupole or a magnetic dipole but not an electric dipole radiation. With equation (4) we
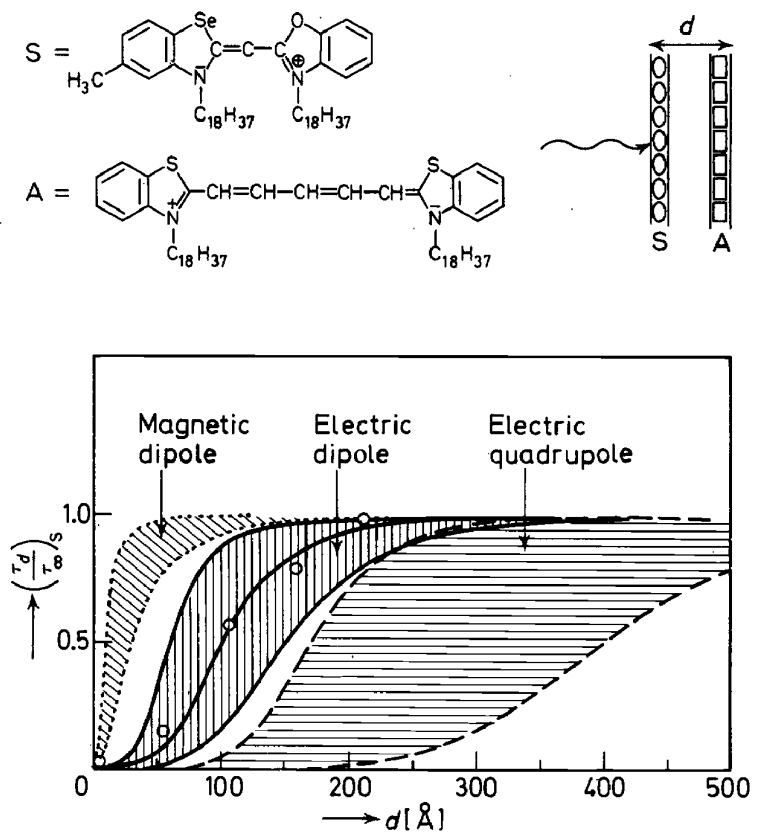

Figure 6. Multipole nature of phosphorescence of dye S. Decay time of phosphorescence $\left(\tau_{d}\right)$ versus distance $d$ between $\mathrm{S}$ and layer of acceptor $\mathrm{A}$. Theoretical range for magnetic dipole, electric dipole and electric quadrupole radiations. Experimental points on theoretical curve for electric dipoles with appropriate value $q_{s}$.

calculate that the quantum yield for an electric dipole radiation lies between the values 0.42 (dipole oscillating parallel to the layer plane) and 0.21 (dipole oscillating perpendicular to the layer plane). Thus the quantum yield $q_{s}$ is distinctly higher than the measured overall quantum yield of the phosphorescence, $q_{s}^{\prime}=0.06$.

The results may be checked by another method (not discussed here) based on the monolayer assembling technique: measuring the directional pattern 
of the phosphorescence as a function of the distance between the emitting molecule and a metal mirror ${ }^{2,10,11}$.

It would be of interest to realize experimentally the quadrupole emitter, since it should result in a considerably larger range of energy transfer than the electric dipole.

\section{(b) Luminescence lifetime in the proximity of an interface}

We have considered the shortening of the lifetime of the excited state by a transfer of energy to an acceptor. A surprising effect may be demonstrated related to energy transfer with the monolayer assembling technique: changing the lifetime of the excited state without changing the luminescence quantum yield ${ }^{12}$. The decay time of the luminescence is found to be dependent on the distance between emitter and and the interface of two differently polarizable dielectrics ${ }^{13}$. It may be shortened or lengthened depending on this distance. Thus the average lifetime of the excited state of a molecule or the probability $\left(\mathrm{d} P_{e}\right)$ of exciting a quantum of light depends on the broad environment of the molecule. The process of emitting the light quantum does not only depend on the excited molecule, but on the total system of molecule plus its environment. The excited molecule, so to say, knows all about its surroundings when it decides to emit a quantum of light. This experiment constitutes an unusual manifestation of the wave-particle duality of light.

For a quantitative consideration it is advantageous to use again the classical picture and equation (1) for translating the result into the quantum mechanical language.

First let us again look at the energy transfer from an excited molecule $S$ described as an oscillating dipole to an acceptor $\mathrm{A}$ in the alternating field of

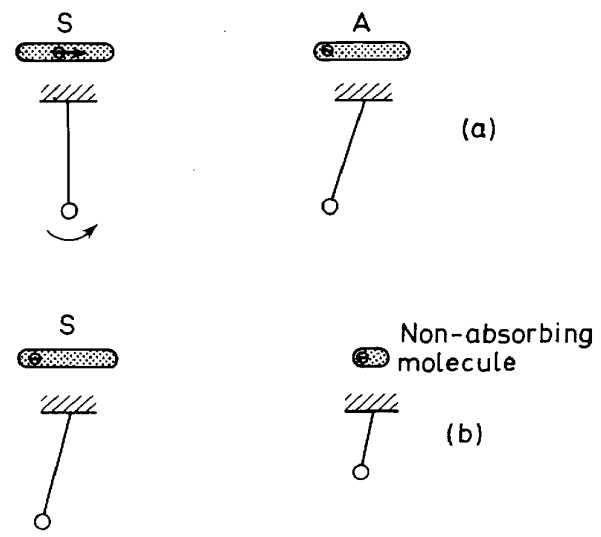

Figure 7. Fluorescent molecule S and acceptor molecule A (a) or non-absorbing molecule (b). Classical picture neglecting retardation effect. Phase shift between oscillators $\mathrm{S}$ and $\mathrm{A}$ in case (a), in-phase oscillation of oscillators in case (b).

$\mathrm{S}$ (Figure 7a). A may be described as a damped oscillator. It will oscillate with a phase shift relative to $S$. The field of $A$ damps $S$ and therefore diminishes the decay time of $S$ : energy is transferred from $S$ to $A$. 


\section{HANS KUHN}

A is now substituted for a non-absorbing molecule. It will oscillate in phase with S, and thus does not damp S (Figure 7b). However, this statement is not completely correct, since the electromagnetic field needs some time to reach the non-absorbing molecule and the echo field to reach $\mathrm{S}$. This echo field has an accelerating or retarding effect on S depending on this phase shift between echo field and elongation of $S$. Thus the molecule, even when not absorbing energy, influences the decay time of $S$.

In the case of an absorbing molecule $\mathrm{A}$, its influence on the emission power $L_{e}$ is negligible and the decrease of the decay time is determined by the absorbed power $L_{a}$. For a non-absorbing molecule, $L_{a}=0$, but the

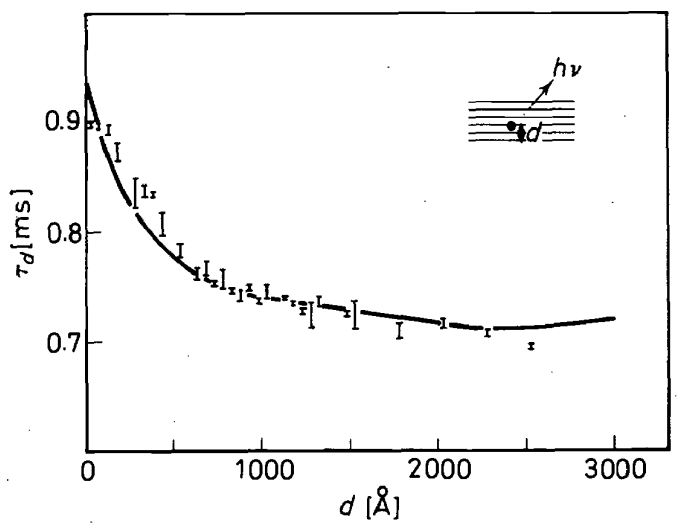

Figure 8. Red luminescent europium complex at distance $d$ from fatty acid/air interface. Lifetime of luminescence $\left(\tau_{d}\right)$ as a function of $d$. Theoretical curve and experimental points. The quantum yield of the luminescence being constant and equal to $1, \tau_{d}$ is markedly dependent on $d$. This effect is caused by the time the electromagnetic radiation needs to travel from the emitter to the neighbouring polarizable molecules and the echo by these molecules to travel to the emitter, i.e. a time of the order of $\left(3 \times 10^{-6} \mathrm{~cm}\right) /\left(3 \times 10^{10} \mathrm{~cm} / \mathrm{sec}\right)=10^{-16} \mathrm{sec}$.

influence of this molecule on the radiative decay process of $\mathrm{S}$ is important. This process then must be considered as being due to the system of both molecules.

In the case of many non-absorbing molecules, a dielectric extending over the shaded part in Figure 8, we have to sum up over the echoes of each molecule and, for a particular case, the curve in Figure 8 is obtained for the decay time $\left(\tau_{d}\right)$ as a function of the distance between emitter and interface $(d)^{13}$. The experimental points correspond to the measured decay times of samples obtained by depositing a monolayer of a strongly luminescent europium complex on a glass plate and superposing on it fatty acid monolayers (fatty acid and glass have almost the same refractive index).

The theoretical curve is obtained by assuming a quantum yield $q_{s}=1$ (this value was obtained by energy transfer experiments ${ }^{2}$ ) and the good agreement between theory and experiment substantiates the value. The resulting theoretical curve depends strongly on $q_{s}$ and thus this type of experiment is useful as a method for determining $q_{s}$, which is otherwise 


\section{CHROMOPHORES IN MONOLAYER ASSEMBLIES}

difficult to determine. Similar experiments with a metal surface as interface have been described elsewhere ${ }^{9,12}$

\section{LIGHT ABSORPTION OF AGGREGATES OF DYES IN MONOLAYER ASSEMBLIES}

\section{(a) Absorption by sandwich-pair aggregates}

In the foregoing part we studied the weak interaction of molecules at relatively large separations. In the following we consider molecules in direct contact. Using the monolayer assembling technique, sandwich-pair arrangements of identical or different dye molecules may be studied ${ }^{14}$. The experimental band shifts and changes in oscillator strength were found to be in quantitative agreement with the values calculated using a refined onedimensional electron gas model ${ }^{15}$. Table 2 gives, for some cases, the theo-

Table 2. Shifts (nm) of some Scheibe-type aggregates $\left(\lambda_{P}\right)$ and dimers $\left(\lambda_{D}\right)$ with respect to the corresponding monomers $\left(\lambda_{M}\right)$

theor. $^{\lambda_{P}-\lambda_{M}}$ exp. theor. ${ }^{\lambda_{D}-\lambda_{M}}$ exp.

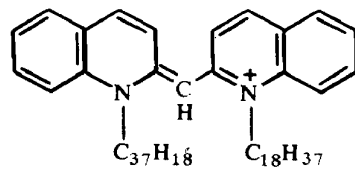

$$
+54
$$
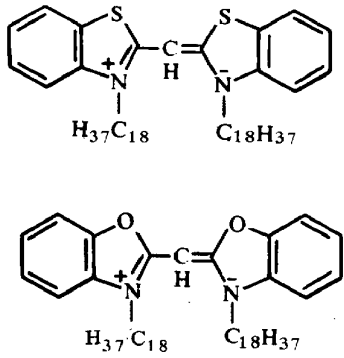

retical and observed shifts $\lambda_{D}-\lambda_{M}$ between the absorption peak of the dimer $\left(\lambda_{D}\right)$ and of the monomer $\left(\lambda_{M}\right)$. Based on this approach is the following assumption which highly facilitates calculation:

The $\sigma$-electrons of the dye molecule and of the surrounding solvent molecules are treated as an infinite medium of dielectric constant 2.5 and the $\pi$-electrons are considered as being in this medium and as moving along the zigzag lines in Figure 9, i.e. along the lines of highest $\pi$-electron density. 


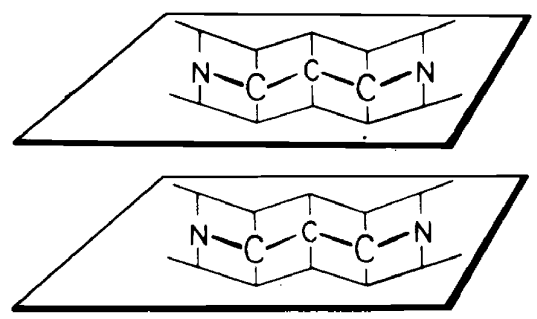

Figure 9. Model used to calculate molecular aggregate spectra shown for simplest cyanine in sandwich arrangement.

\section{(b) Absorption by Scheibe-dye aggregates}

This model may be applied to the Scheibe-dye aggregates (J-aggregates) ${ }^{16}$ characterized by a very narrow and high absorption peak corresponding to an in-phase oscillation of the oscillators substituting each dye molecule ${ }^{17}$. In the well known case of pseudo-isocyanine (Figure 10) this peak has a bathochromic shift of $53 \mathrm{~nm}$ relative to the monomer peak.

The aggregate structures shown in Figures $10 a$ and $b$ have been proposed<smiles>CCN1C(=Cc2ccc3ccccc3[n+]2CC)C=Cc2ccccc21</smiles>

$$
\begin{aligned}
\lambda_{P}-\lambda_{M}[\mathrm{~nm}]= & +53 \\
& \text { (experimental) }
\end{aligned}
$$

a)

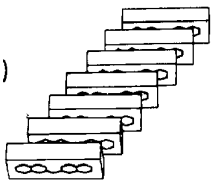

b)

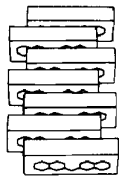

c)

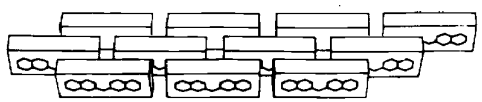

$+44$

$\lambda_{P}-\lambda_{M}[\mathrm{~nm}]=-40$

$-52$

Figure 10. Scheibe-aggregate forming dye pseudoisocyanine. Experimental and theoretical values for aggregate to monomer band shift $\lambda_{P}-\lambda_{M}$. (a) staircase arrangement; (b) ladder arrangement; (c) brick-stonework arrangement.

in the past ${ }^{16}$. Assuming these structures, the electron gas model calculation gives hypsochromic instead of bathochromic shifts ${ }^{18}$. However, when the structure shown in Figure 10c (a brick-stonework-like arrangement of the dye molecules) is assumed the model calculation gives a bathochromic shift of $44 \mathrm{~nm}$, which is in approximate agreement with experiment. It is a well known fact that the formation of Scheibe-dye aggregates is facilitated by bulky meso-substituents. This fact is explained by the brick-stonework model, which allows a tight packing of the molecules.

Keeping in mind this new structural concept, let us try to find a way to get Scheibe-aggregates of dyes by appropriately arranging the molecules in 


\section{CHROMOPHORES IN MONOLAYER ASSEMBLIES}

the monolayer ${ }^{19}$. We consider stearyl substituted cyanine dyes, not known as aggregate forming, such as dye $\mathrm{S}$ in Figure 1. The chromophore may be viewed schematically as a rectangular block with the dimensions given in Figure $11 a$, and the two hydrocarbon substituents as cylinders of diameter $5 \AA$. According to Figure $11 b$ a close packing of the chromophores is only possible for a brick-stonework-like arrangement, with the chromophore

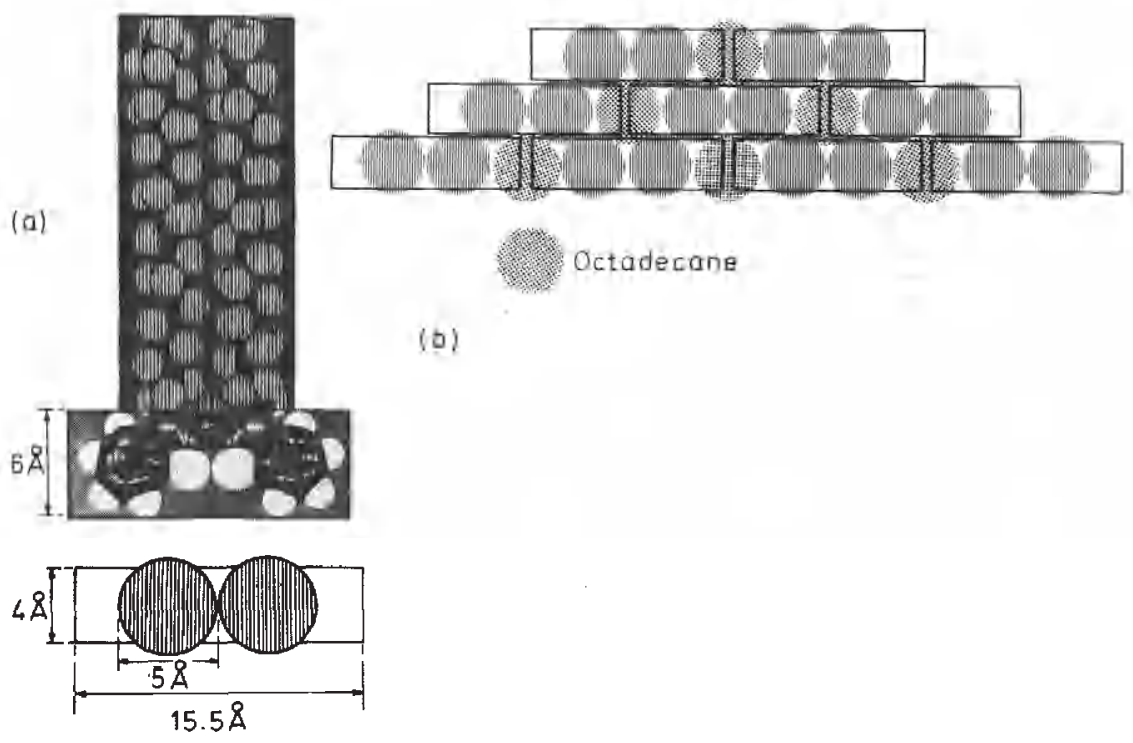

Figure 11. Scheibe-dye-aggregate formation in monolayer. (a) Dye molecule in broad side view and bird's-eye view. Chromophores approximated by rectangular block, stearyl groups by cylinders. (b) Brick-stonework arrangement of chromophores, octadecane chains fitting in the cylindrical holes left over by the stearyl substituents of the dye.

planes perpendicular to the monolayer plane, and the chromophore long axes parallel. This arrangement should lead to a stable layer, if closest packing of the hydrocarbon chains is also achieved. This condition is met by putting a hydrocarbon chain into each hole of the structure left over by the stearyl substituents of the dye molecules, i.e. by spreading a $1: 1$ mixture of this dye and a hydrocarbon such as octadecane (Figure 11b). The brickstonework aggregates are forced to be formed by this trick and are really observed $^{19}$ : the typical narrow absorption band and the almost coinciding fluorescence band occur (Figure 12). The band, as explained by the model, appears only within a small range of the dye to hydrocarbon mixing ratio. For mixed dye monolayers, with a mixing component having a polar head group, the Scheibe-dye aggregate peak is not observed (Figure 12b): the polar head group of the mixing component breaks a hole in the structure.

In pure dye monolayers no compact structure is possible and no Scheibeaggregates are observed. Table 2 shows, for some cases. the theoretical and 


\section{HANS KUHN}

experimental shift $\lambda_{P}-\lambda_{M}$ of the peak of the Scheibe-dye-aggregates $\left(\lambda_{P}\right)$ with respect to that of the monomer $\left(\lambda_{M}\right)$.

In the case of carbocyanine dyes, which have two additional methine groups in the chain, a closely packed structure of mixed monolayers of dye

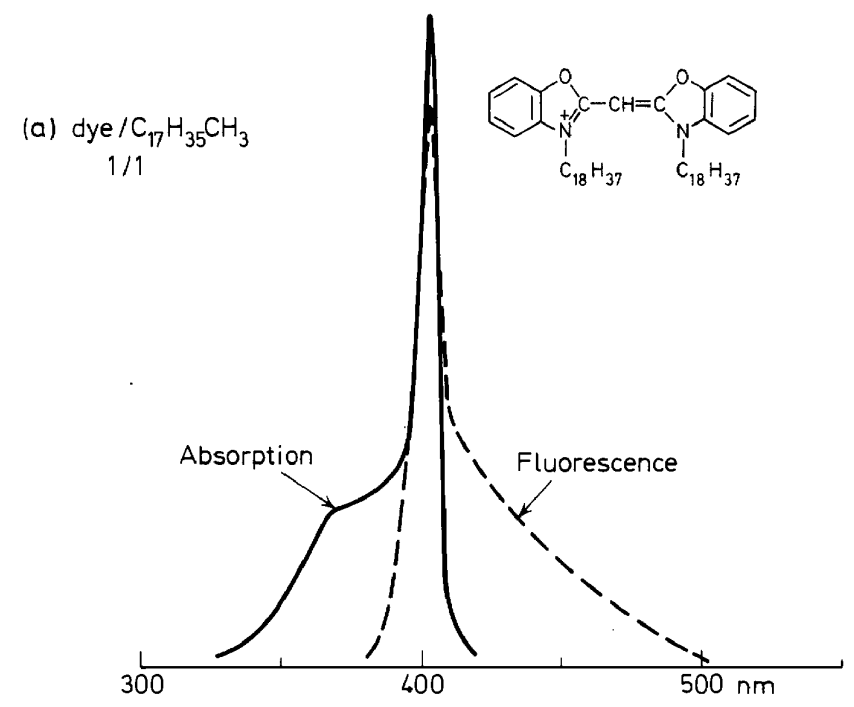

(b) dye $/ \mathrm{C}_{17} \mathrm{H}_{35} \mathrm{COOH}$

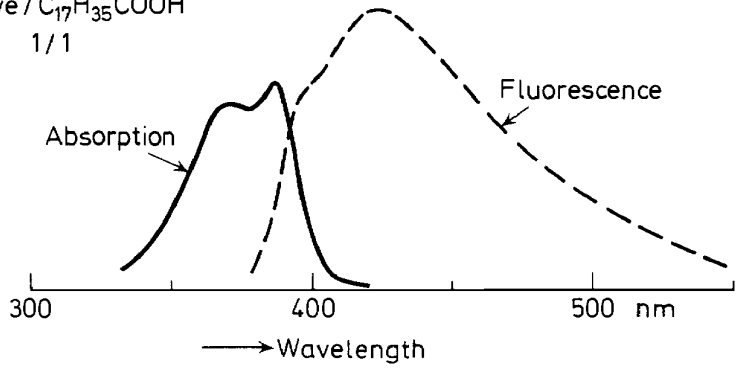

Figure 12. Spectra of cyanine dye and octadecane (a) and arachidic acid (b) in mixing ratio $1: 1$.

and octadecane is not possible and we may conclude that these dyes, contrary to the dyes considered above, should form no Scheibe-aggregates under similar conditions, and no such aggregates have been observed.

\section{(c) Electrochromism of Scheibe-dye aggregates}

A brick-stonework aggregate monolayer may be sandwiched between arachidic acid monolayers and semitransmitting aluminium layers (Figure $13 a)^{20}$. By applying voltages of a few volts, electric fields up to $5 \times 10^{6} \mathrm{~V} / \mathrm{cm}$ may be applied to the dye molecules. The excitation energy of the dye is changed in such fields and shifts of the absorption band are observed. 
A shift proportional to the applied field and a contribution proportional to the square of the field may be distinguished. The first contribution measures the difference between the dipole moment of the excited state and the ground state of the molecule, the second the change in polarizibility.

The band shift is obtained by measuring the absorption change as a function of the frequency of the incident light (Figure 14). (In order to ensure a simple situation it is necessary to keep the distance between the aluminium electrodes such that the system has the property of an interference filter). In the example of Figure 14 the values $\Delta \mu_{y}=0.07$ Debye and $\Delta \alpha_{y y}=1.5 \AA^{3}$ are obtained from the linear and quadratic contributions, respectively.

In the known methods for obtaining information on these quantities, in contrast to the present method, the molecules are oriented in an electric field $^{21}$. Then both polarizibility and dipole moment changes produce band

(a)

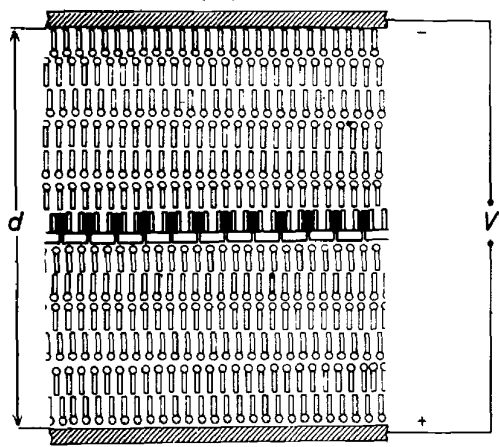

$[\beta$ Surfaceactive cyanine dye

$\infty$ Cd-arachidate - Octadecane

vellot Semitransparent Al-electrodes (b)

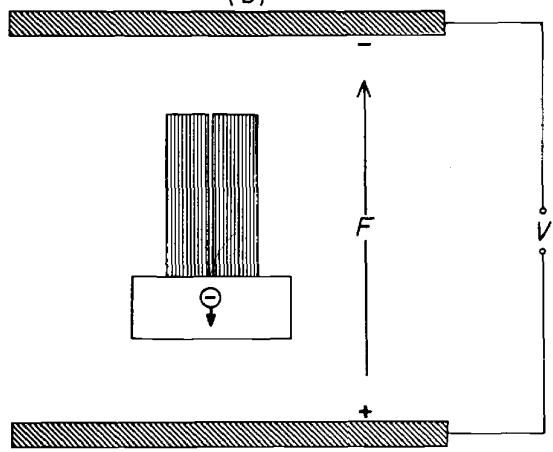

Figure 13. (a) Cross section through capacitor for measuring electrochromism. A single monolayer of Scheibe-dye-aggregate forming cyanine sandwiched between monolayers of cadmium arachidate and semitransparent electrodes. (b) Dye molecule in electric field. When light is absorbed negative charge moves in direction of arrow.

shifts proportional to the square of the field. For this reason it is not possible to measure polarizibility changes by these methods.

Theoretical values for $\Delta \mu_{y}$ and $\Delta \alpha_{y y}$ of the dye in Figure 14, obtained by an electron gas model calculation $\left(\Delta \mu_{y}=0.2\right.$ Debye, $\left.\Delta \alpha_{y y}=3 \AA^{3}\right)$, have the same sign and similar absolute values as the above experimental data.

In the case of the linear contribution the essentials of the calculation are seen by considering the electron clouds given by the electron gas model in its simplest form, neglecting the branching of the $\pi$-electron system. The chromophore system then extends over the shaded area in Figure 15. The wave function of the highest occupied state has a wave with three antinodes, the next state four antinodes. By inspecting the electron clouds in both states it is immediately seen that the centre of gravity of the cloud, when exciting the chromophore, is shifted in the direction of the arrow in Figure $13 b$. 


\section{HANS KUHN}

In the presence of an electric field, with a direction as indicated in Figure $13 b$, the excitation energy $\Delta E$ is decreased, since the excitation of the molecule moves negative charge towards the positive electrode. Thus the absorption band of each single molecule is shifted to the red when applying the voltage. This causes a corresponding shift of the aggregate band: in the

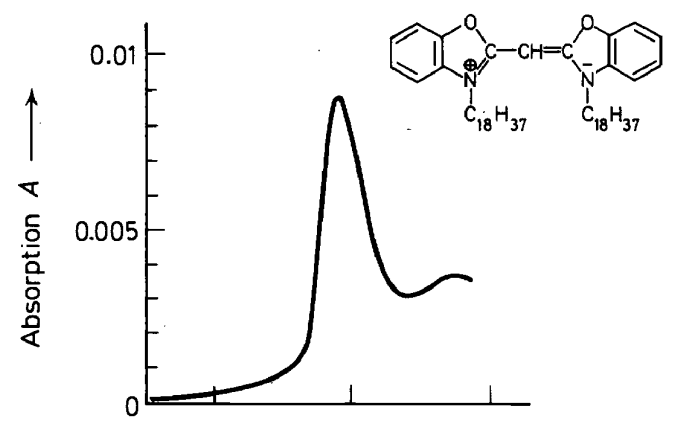

(a)

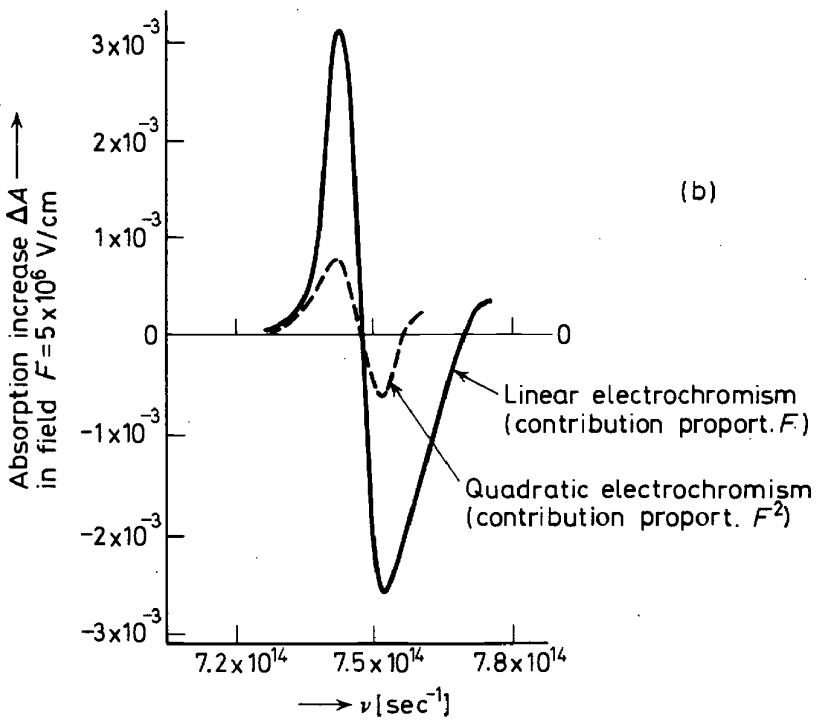

Figure 14. Electrochromism of cyanine dye. (a) Absorption $A(v)$ of monolayer on a glass plate ; (b) Monolayer in condenser of Figure 13. Absorption change $\Delta A(v)$ induced in electric field of $5 \times 10^{6} \mathrm{~V} / \mathrm{cm}$. Contribution proportional to $F$ (full curve); contribution proportional to $F^{2}$ (dashed curve).

classical picture the dipole substituting the dye oscillates slower and, consequently, the frequency of the coupled in-phase oscillation corresponding to the aggregate band is slower. When the polarizability of the applied voltage is reversed, the excitation energy is increased. 


\section{CHROMOPHORES IN MONOLAYER ASSEMBLIES}
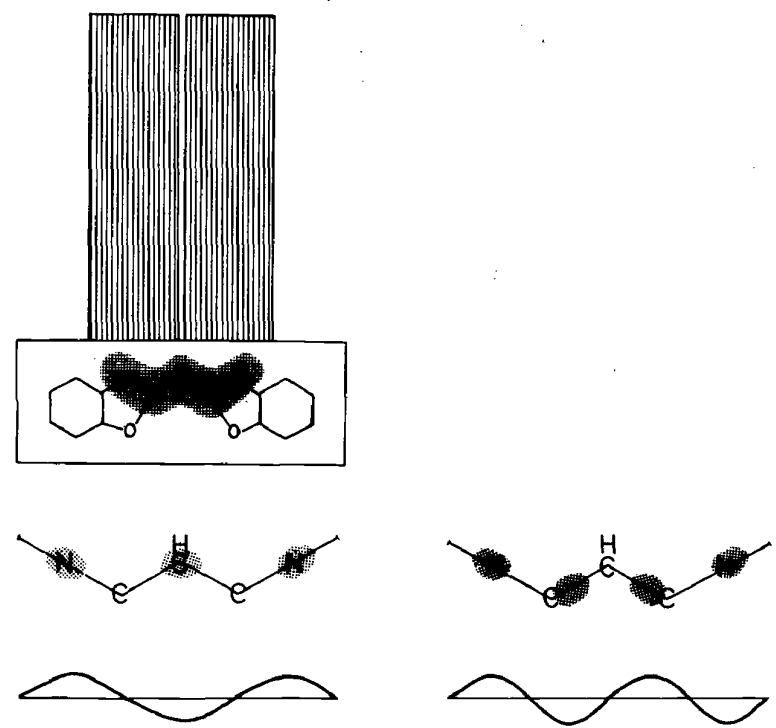

Figure 15. Dye in Figure 14. Portion of chromophore taken into account in model calculation (shaded area). Proceeding from highest occupied orbital (three antinodes) to next orbital (four antinodes), the centre of gravity of electron cloud moves in direction from top to bottom.

\section{REFERENCES}

${ }^{1}$ M. M. Zwick and H. Kuhn. Z. Naturforsch. 17a, 411 (1962); K. H. Drexhage, M. M. Zwick and H. Kuhn. Ber. Bunsenges. Phys. Chem. 67, 62 (1963).

2 H. Bücher, K. H. Drexhage, M. Fleck, H. Kuhn, D. Möbius, F. P. Schäfer, J. Sondermann, W. Sperling, P. Tillmann and J. Wiegand. Mol. Cryst. 2, 199 (1967).

H. Bücher, H. Kuhn, B. Mann, D. Möbius, L. v. Szentpály and P. Tillmann. Photogr. Sci. Engng. 11, 233 (1967); Equation (8) should read $E=K \Delta(\ln 10)^{-1}$ and in the equation following (8), instead of $\alpha=(3 / 4 \pi)[(\ln ) / 24]^{\ddagger}=0.133, \operatorname{read} \alpha=(3 / 4 \pi)[(\ln 10) 36]^{\ddagger}=0.120$.

H. Bücher, O. v. Elsner, D. Möbius, P. Tillmann and J. Wiegand. Z. Physik. Chem. N. F. 65, 152 (1969).

${ }^{3}$ O. Inacker, D. Möbius and H. Kuhn. In print.

4 A. Rothen. J. Phys. Chem. 63, 1929 (1959); Biochim. Biophys. Acta 88, 606 (1964): Physical Techniques in Biological Research D. H. Moore, Ed., Vol. II, Part A, 2nd ed., Academic Press, New York and London, 1968, p. 217.

${ }^{5}$ W: Mormann and H. Kuhn. Z. Naturforsch. 24b, 1340 (1969).

6 P. Mueller, D. O. Rudin, H. T. Tien and W. C. Wescott in Recent Progress in Surface Science Vol. I. Academic Press, New York, London 1964, Ch. II.

7 P. Fromherz. FEBS Letters, 11, 205 (1970); Nature, Lond. 231, 267 (1971); Biochim. Biophys. Acta, 225, 382 (1971).

${ }^{8}$ S. Freed and S. I. Weissmann. Phys. Rev. 60, 440 (1941); S. I. Weissmann and D. Lipkin. J. Am. Chern. Soc. 64, 1916 (1942).

9 H. Kuhn, J. Chem. Phys. 53. 101 (1970).

10 O. Inacker, H. Kuhn, H. Bücher, H. Meyer and K. H. Tews. Chem. Phys. Lett. 7, 213 (1970). Instead of $q_{s}=0.14$ in first line of left row on page 215 , read $q_{s}=0.41$.

${ }^{11}$ M. Fleck. Thesis, University of Marburg, 1968; K. H. Drexhage, M. Fleck and H. Kuhn. Ber. Bunsenges. Phys. Chem. 71, 915 (1967).

${ }^{12}$ K. H. Drexhage, M. Fleck, H. Kuhn, F. P. Schäfer and W. Sperling. Ber. Bunsenges. Phys. Chem. 70,1179 (1966); K. H. Drexhage, H. Kuhn, F. P. Schäfer. Ber. Bunsenges. Phys: Chem. 72, $329(1968)$. 


\section{HANS KUHN}

${ }^{13}$ K. H. Tews, O. Inacker and H. Kuhn. Nature 909, 276 (1970). Equation (5) contains a printing error. Instead of $x(\sin (x)-\pi / 2)$ read: $x(\operatorname{Si}(x)-\pi / 2)$; instead of 'ref. 5 ' in the third paragraph read 'ref. 6'.

14 H. Kuhn. Naturwissenschaften 54, 429 (1967).

15 V. Czikkely, G. Dreizler, H. D. Försterling, H. Kuhn, J. Sondermann, P. Tillmann and J. Wiegand. Z. Naturforsch. 24a, 1821 (1969).

16 G. Scheibe. Angew. Chem. 52, 633 (1939); O. Wörz and G. Scheibe. Z. Naturforsch. 24h, 381 (1969).

17 Th. Förster. Naturwissenschaften 33, 166 (1946); E. G. McRae and M. Kasha. J. Chem. Phys. 28, 721 (1958).

18 V. Czikkely, H. D. Försterling and H. Kuhn. Chem. Phys. Lett. 6, 11 (1970).

19 H. Bücher and H. Kuhn. Chem. Phys. Lett. 6, 183 (1970).

${ }^{20}$ H. Bücher and H. Kuhn. Z. Naturforsch. 25b, 1323 (1970).

${ }^{21}$ See e.g. W. Liptay. Angew. Chem. 81, 195 (1969); K. Siebold, H. Navangul and H. Labhardt. Chem. Phys. Lett. 3, 275 (1969). 